\title{
Distribution et dégâts associés au thrips de l'oignon, Thrips tabaci L. (Thysanoptera : Thripidae) en fonction de la zone agro-climatique au Burkina Faso
}

\author{
Abdoulaye SAVADOGO ${ }^{1}$, Boniface Babo BAKOUAN ${ }^{1}$, Mathieu Wendnéyidé \\ SAWADOGO ${ }^{3}$, Karim NÉBIÉ2 2 Rémy DABIRÉ2 ${ }^{2}$ Djakaria SON${ }^{3}$, Irénée SOMDA ${ }^{3}$, \\ Schémaeza BONZI ${ }^{3}$, Gaston DABIRÉ ${ }^{4}$, Hyacinthe KAMBIRÉ ${ }^{5}$, Anne LEGRÈVE ${ }^{6}$, \\ François J. VERHEGGEN ${ }^{7}$ et Souleymane NACRO ${ }^{5 *}$
}

\author{
${ }^{1}$ Centre Agricole Polyvalent de Matourkou, 01 BP 130 Bobo-Dioulasso 01, Burkina Faso. \\ ${ }^{2}$ Centre Régional de Recherches Environnementales et Agricoles de l'Ouest, 01 BP 910 Bobo-Dioulasso 01, \\ Burkina Faso. \\ ${ }^{3}$ Institut du Développement Rural, Université Nazi Boni, 01BP1091 Bobo-Dioulasso 01, Burkina Faso. \\ ${ }^{4}$ Centre Universitaire Polytechnique de Gaoua. 01 BP1091 Bobo-Dioulasso 01 Burkina Faso. \\ ${ }^{5}$ Centre Régional de Recherches Environnementales, Agricoles et de Formation de Kamboinsé, 01 BP 476 \\ Ouagadougou 01, Burkina Faso. \\ ${ }^{6}$ Earth and Life Institute, Catholic University of Louvain, Louvain la Neuve, Belgium. \\ ${ }^{7}$ Gembloux Agro-Bio Tech, TERRA, University of Liege, Gembloux, Belgium. \\ *Auteur correspondant ; E-mail : snacro2006@yahoo.fr
}

\section{RESUME}

L'oignon est le premier légume produit au Burkina Faso. Thrips tabaci constitue son principal insecte ravageur. Ce travail avait pour objectif d'évaluer la distribution, l'incidence et la sévérité des attaques de $T$. tabaci sur l'oignon. L'étude a été réalisée dans trois bassins de production représentatifs de deux zones agroécologiques du Burkina Faso: la zone sahélienne caractérisée par une pluviosité moyenne annuelle inférieure à $600 \mathrm{~mm}$ et une température moyenne annuelle de $35^{\circ} \mathrm{C}$; contre une pluviosité moyenne annuelle comprise entre 600 et $900 \mathrm{~mm}$ et une température moyenne annuelle de $33{ }^{\circ} \mathrm{C}$ pour la zone nord soudanienne Nous avons réalisé des prospections dans 45 parcelles paysannes dans les zones de production ciblées. Des plantes d'oignon ont été prélevées dans trois quadrats posés sur une des diagonales de chaque parcelle, et les thrips trouvés sur chaque plante de chaque quadrat comptés. Tous les plants à l'intérieur du quadrat ont été donc observés. La densité moyenne la plus élevée $\left(216 \pm 385\right.$ thrips au $\left.\mathrm{m}^{2}\right)$ a été observée dans la région du Nord tandis que l'incidence moyenne la plus élevée $(95,91 \pm 8,92 \%)$ a été observée dans la Boucle du Mouhoun. La plus faible sévérité $(19,29 \pm 13,27 \%)$ a été enregistrée dans le Plateau central. Cette étude est un premier pas vers le développement de stratégies alternatives de lutte contre les parasites dans le cadre de systèmes de production végétale durables à l'aide de bonnes pratiques agricoles et de l'utilisation des pesticides biologiques.

(C) 2020 International Formulae Group. All rights reserved.

Mots clés : Oignon, Thrips tabaci, incidence, sévérité, répartition, Burkina Faso. 


\title{
Distribution and damage associated with the onion thrips, Thrips tabaci L. (Thysanoptera: Thripidae) according to the agro-climatic zone in Burkina Faso
}

\begin{abstract}
Onion is the first vegetable produced in Burkina Faso. Thrips tabaci is its main insect pest. We assessed the distribution, incidence and severity of $T$. tabaci attacks on onion in three-production areas representative of two agroecological zones of Burkina Faso: the the Sahelian zone characterized by an average annual rainfall of less than $600 \mathrm{~mm}$ and an average annual temperature of $35^{\circ} \mathrm{C}$; compared to an average annual rainfall of between 600 and $900 \mathrm{~mm}$ and an average annual temperature of $33{ }^{\circ} \mathrm{C}$ for the northern Sudanian zone. We carried out surveys in 45 farmer plots in the targeted production zones. Thus, onion plants were taken from three quadrats placed on one of the diagonals of each plot, and the thrips found on each plant were counted. All plants within the quadrat were therefore observed. The highest average density $\left(216 \pm 385\right.$ thrips per $\left.\mathrm{m}^{2}\right)$ was observed in the North while the highest average incidence $(95.91 \pm 8.92 \%)$ was observed in the Boucle du Mouhoun. The lowest severity $(19.29 \pm 13.27 \%)$ was recorded in the Plateau central region. This study is a first step towards the development of alternative pest control strategies within sustainable crop production systems using good agricultural practices and the use of biopesticides.

(C) 2020 International Formulae Group. All rights reserved.
\end{abstract}

Keywords: Onion, Thrips tabaci, incidence, severity, distribution, Burkina Faso.

\section{INTRODUCTION}

Le maraîchage est pratiqué dans toutes les régions du Burkina Faso. Le nombre de sites maraîchers était estimé en 2010 à 4844 à travers le pays (DPSSA, 2011).

L'oignon (Allium cepa) est la première culture maraichère du Burkina Faso (PDCFL, 2017) tant du point de vue des superficies exploitées que des quantités produites. En 2010, 11449 ha étaient emblavés au niveau national soit $41,4 \%$ de la superficie totale des productions maraîchères (DPSSA, 2011). Cinq régions enregistraient chacune plus de 1000 ha de superficies emblavées en oignon bulbe : le Nord (3 165 ha), le Centre-Ouest (1 662 ha), les Hauts-Bassins ( 1423 ha), le Centre-Nord (1 165 ha), et la Boucle du Mouhoun (1 039 ha). Selon la même source, la production totale d'oignon bulbe était de 242258 tonnes soit $32,4 \%$ de la production maraîchère totale. Cinq régions ont contribué pour $71 \%$ à la production nationale. Il s'agit des régions du Nord (65 384 tonnes), des Hauts-Bassins (29 968 tonnes), de la Boucle du Mouhoun (27 049 tonnes), du Centre-Ouest (26 725 tonnes), et du Plateau Central (22 538 tonnes).
Les revenus apportés par la production de l'oignon bulbe en 2010 étaient estimés à 24,87 milliards FCFA, soit $30 \%$ de la valeur totale des ventes des produits maraichers (DPSSA, 2011). La culture de l'oignon est soumise à de nombreuses contraintes comme les maladies, les insectes ravageurs, les nématodes etc. Parmi ces ravageurs, le thrips de l'oignon est sans doute le plus redoutable (Leblanc, 2010). Il entraine la destruction du feuillage par le prélèvement de sa nourriture et la transmission de virose avec des répercussions sur la croissance et la maturation de l'oignon (Biao et al., 2019). Ces dommages peuvent entraîner des pertes de rendement pouvant aller jusqu'à $60 \%$ (Cliniques des plantes, 2018). Si l'attaque est précoce, toute la culture peut être détruite (Anonyme, 2019). Le thrips est donc le principal ravageur de l'oignon et est très difficile à combattre (Leblanc, 2010). En plus de l'oignon, il attaque le niébé, le tabac etc. (Liao et Lin, 2000); Egho,2010 et Issoufou et al., 2017).

Peu d'études sur le thrips ont menées au Burkina Faso alors que ce ravageur constitue une sérieuse menace pour l'oignon, principale culture maraîchère du pays. C'est ce constat 
qui justifie la présente étude dont l'objectif est d'évaluer la distribution, l'incidence et la sévérité des attaques de $T$. tabaci sur l'oignon.

\section{MATERIEL ET METHODES}

\section{Présentation de la zone d'étude}

L'étude a été menée dans les régions de la Boucle du Mouhoun, du Plateau Central et du Nord qui sont les principales zones de production d'oignon au Burkina Faso. Les communes qui polarisent l'étude sont Loumbila (province de l'Oubritenga dans le Plateau Central), Ouahigouya et Namissiguima, (province du Yatenga dans la région du Nord) et Bourasso et Sonon (province de la Kossi dans la Boucle du Mouhoun).

La province du Yatenga appartient à la zone sahélienne; celles de Kossi et de l'Oubritenga appartiennent à la zone nord soudanienne (Figure 1). La province du Yatenga a enregistré une pluviosité de 219,7 mm en 44 jours de pluie en 2017 contre 860 $\mathrm{mm}$ en 49 jours en 2018. Une pluviosité de $667,8 \mathrm{~mm}$ en 35 jours a été enregistrée dans la Kossi en 2017 contre 716,19 mm en 49 jours en 2018 (DPAAH/Kossi). En 2017, 712,97 mm en 37 jours ont été enregistrés dans l'Oubritenga contre $753,25 \mathrm{~mm}$ en 48 jours en 2018 (DRAAH/Plateau central).

\section{Echantillonage et évaluation des dégâts des thrips}

Dans les trois régions de l'étude, les données sur les populations et les dégâts des thrips ont été collectées dans 45 parcelles au total, soit cinq parcelles par site. Parmi les dix producteurs retenus par site pour les enquêtes, cinq producteurs d'oignon ont été choisis pour la collecte des données sur les populations et les dégâts des thrips. Sur chaque parcelle retenue pour la prospection, trois quadrats d'un mètre carré chacun ont été posés sur une des diagonales de la parcelle. Dans chaque quadrat, cinq plants d'oignon ont été prélevées, soit au total 15 plants par parcelle. Chaque plant a été isolé dans un sachet plastique transparent pour des observations futures. Les cinq sachets contenant les plantes prélevées ont été gardés dans une enveloppe de papier kraft confectionnée à cet effet. Sur cette enveloppe sont inscrits le numéro de la parcelle, celui du quadrat et le nom du site. Sur une fiche de prospection préétablie, l'identité du producteur, le nom du site, les coordonnées géographiques et le numéro de la parcelle sont inscrits.

\section{Evaluation de l'incidence des attaques des thrips sur l'oignon}

Pour pouvoir évaluer l'incidence des attaques des thrips sur l'oignon, tous les plants d'oignon du quadrat ont été comptées. Après ce comptage, les plants du même quadrat ayant des symptômes des attaques des thrips ont aussi été comptées.

L'incidence des attaques de thrips a été évaluée à partir de la formule suivante :

$$
\mathbf{I}(\%)=(\mathbf{n} / \mathbf{N}) \times \mathbf{1 0 0} \text {. Où }
$$

$\mathbf{n}=$ nombre des plants du quadrat attaquées par les thrips;

$\mathbf{N}=$ nombre total de plants du quadrat ;

$\mathbf{I}=$ incidence des attaques des thrips du quadrat.

L'incidence des attaques des thrips de la parcelle est obtenue en calculant la moyenne des incidences des trois quadrats. La même procédure a été utilisée pour obtenir l'incidence du site et celle du bassin de production.

\section{Evaluation de la distribution des populations de thrips}

Les plants prélevés ont été emmenés dans les bureaux des antennes régionales de la clinique des plantes pour le comptage des thrips. Chaque plante a été bien secouée sur une feuille de papier blanc ou sur un plateau et ses feuilles séparées afin de recueillir tous les thrips présents sur la plante. Les larves et les adultes des thrips de chaque plante ont été comptés et le nombre inscrit sur la fiche de prospection. Le comptage des thrips a été réalisé à l'aide d'une loupe et d'un compteur manuels. Les densités des thrips ont été calculées à partir des formules suivantes : 
- La densité moyenne des populations de thrips par unité de surface $\left(\mathrm{m}^{2}\right)$ de la parcelle $=($ somme des adultes + larves des trois quadrats) $/ 3$;

- La densité moyenne des populations de thrips du site $=$ (densité moyenne des populations de thrips des parcelles) / 5 (nombre de parcelles) ;

- La densité moyenne des populations de thrips du bassin $=$ (la densité moyenne des populations de thrips des sites) / 3 (nombre de sites).

\section{Evaluation de la sévérité des attaques des thrips sur l'oignon}

La sévérité des attaques des thrips a été évaluée en utilisant l'échelle Ctifl (Centre technique interprofessionnel des fruits et légumes) et l'échelle de notation des symptômes de l'alternariose (Ablo-Elyours, 2014 ; Dabiré, 2016) adaptées aux attaques des thrips.

Les cinq plants d'oignon prélevés dans le quadrat ont été utilisées pour évaluer la sévérité des attaques des thrips. Chacune des feuilles des plants prélevés a été observée et classée sur la base des symptômes des attaques des thrips sur celle-ci. Cette classification a été faite à l'aide des échelles Ctifl et de AboElyours (Figure 2).

La sévérité des attaques des thrips sur l'oignon a été calculée par la formule suivante : Sévérité des attaques $(\%)=($ Somme des différentes notes des feuilles infestées $\times 100)$ / (Nombre total de feuilles $\times 4$ ) (Abo-Elyours, 2014 ; Dabiré, 2016).

\section{Analyse et traitement des données}

Les données collectées sur la distribution des thrips et de leurs dégâts ont été saisies, regroupées et analysées à l'aide du tableur Microsoft Excel 2016. Les analyses ont porté sur les statistiques descriptives : pourcentages, moyennes, écarts-types et traitements graphiques. L'analyse de variance a été réalisée avec le logiciel Statistix 10.0 et la séparation des moyennes a été faite avec le test de Student-Newman-Keuls (S-N-K) au seuil de $5 \%$.

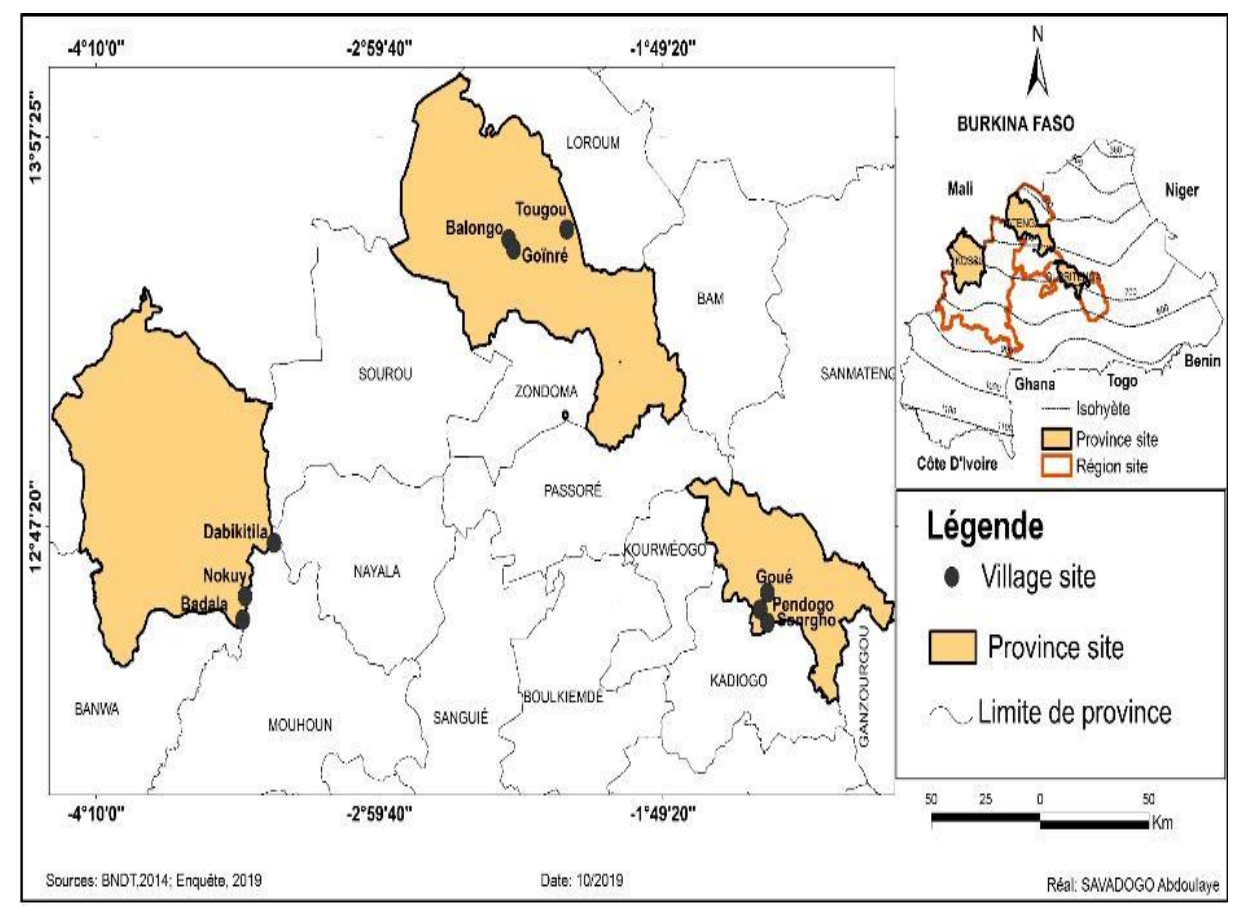

Figure 1: Situation géographique des sites couverts par l'étude. 


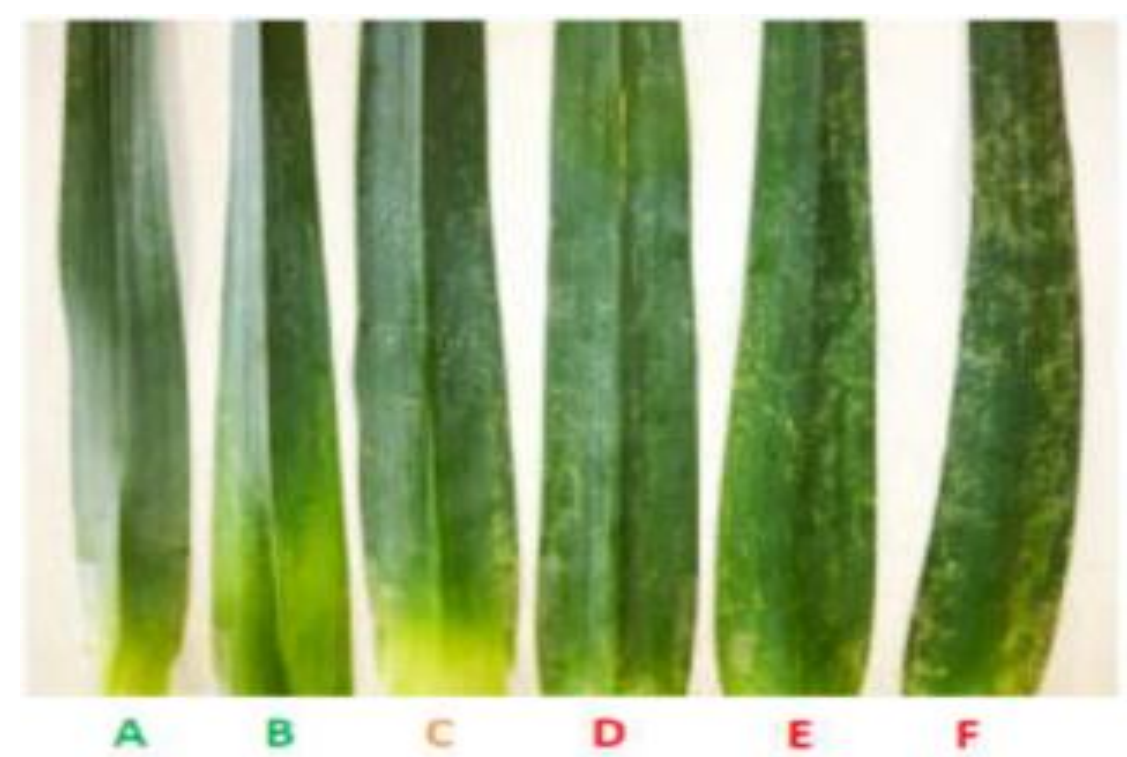

Figure 2: Echelle Ctifl/Classes de dégâts sur feuillage (Source : Sileban, 2014).

A et B : Feuille saine ou sans taches (Note 0); C : Taches sur 10 à 20\% de la feuille (Note 1); D : Taches sur 30 à $40 \%$ de la feuille (Note 2); E : Taches sur 50 à $60 \%$ de la feuille (Note 3); F : Taches sur plus de $60 \%$ de la feuille (Note 4).

\section{RESULTATS}

Evaluation de la densité des thrips, de l'incidence et de la sévérité en fonction de la zone agroécologique

Le Tableau 1 illustre la densité moyenne des thrips au $\mathrm{m}^{2}$, l'incidence et la sévérité moyennes des attaques en fonction de la zone agro climatique. L'analyse de variance a révélé une différence significative entre les deux zones agro climatiques en termes de densité moyenne des thrips au $\mathrm{m}^{2}(\mathrm{P}<0,037)$. La densité la plus élevée a été enregistrée dans la zone sahélienne avec $216 \pm 234$ thrips au $\mathrm{m}^{2}$. La plus faible $\left(64 \pm 165\right.$ thrips au $\left.\mathrm{m}^{2}\right)$ a été enregistrée dans la zone nord soudanienne. Quant aux autres paramètres (incidence et sévérité moyennes) l'analyse de variance n'a pas révélé de différence significative entre les deux zones agro climatiques couvertes par l'étude.

\section{Densité des thrips en fonction des différents bassins et sites de production}

La densité moyenne de thrips au $\mathrm{m}^{2}$ en fonction du bassin de production est illustrée par la Figure 3. Cette variable différait significativement $(P=0,1014)$ entre la région du Nord $\left(216 \pm 385\right.$ thrips au $\left.\mathrm{m}^{2}\right)$ et celle du Plateau central $\left(42 \pm 34\right.$ thrips au $\left.\mathrm{m}^{2}\right)$. Celle de la Boucle du Mouhoun $\left(86 \pm 64\right.$ thrips au $\left.\mathrm{m}^{2}\right)$ ne différait pas de celle des deux autres régions.

La Figure 4 illustre la densité moyenne de thrips au $\mathrm{m}^{2}$ en fonction du site de production. La densité moyenne de thrips la plus élevée a été observée à Balongo (région du nord) avec $461 \pm 618$ thrips au $\mathrm{m}^{2}$. Elle ne différait pas d'avec celle des autres sites où on a noté des densités moyennes de $147 \pm 73$ et 129 \pm 119 thrips au $\mathrm{m}^{2}$ à Nokuy et à Goïnré respectivement, $9 \pm 9$ thrips au $\mathrm{m}^{2}$ à Goué et entre $51 \pm 33$ et $62 \pm 34$ thrips au $\mathrm{m}^{2}$ dans les autres sites (Figure 4) $(\mathrm{P}=0,0000)$.

\section{Niveau d'incidence des attaques des thrips en fonction des différents bassins et sites de production}

Le niveau moyen de l'incidence des attaques des thrips en fonction du bassin de production est illustré par la Figure 5. Le niveau moyen de l'incidence des attaques des thrips le plus bas a été observé dans la région du Plateau central avec $31,31 \pm 13,27 \%$ des plants attaquées par les thrips. Le niveau le plus élevé a été observé dans la région de la Boucle 
du Mouhoun avec 95,91 $\pm 8,92 \%$ des plants attaquées par les thrips. La région du Nord a enregistré une incidence intermédiaire $(72,33 \pm$ $39,29 \%)(\mathrm{P}=0,0000)$.

La Figure 6 illustre les incidences moyennes des attaques des thrips en fonction du site de production d'oignon. L'incidence moyenne la plus faible a été enregistrée sur les sites de Tougou (20,49 $\pm 17,20 \%)$, Goué $(22,38$ $\pm 11,62 \%)$ et Pendogo $(28,57 \pm 13,05 \%)$ qui ne différaient pas entre elles. Le site de Sonrgho $(42,99 \pm 5,6 \%)$ avait une incidence moyenne intermédiaire entre les sites qui enregistraient la plus faible incidence et le groupe qui avait la plus grande incidence dont le site de Balongo, Dabokitila, Nokuy avec $100 \%$ d'incidence, Goinré $(96,5 \pm 6,7 \%)$ et Badala $(87,74 \pm$ $12,4 \%)$.

\section{Niveau de sévérité des attaques des thrips en fonction des différents sites et bassins de production}

La sévérité moyenne des attaques de $T$. tabaci en fonction du bassin de production est illustrée par la Figure 7. La sévérité moyenne la plus faible $(19,29 \pm 9,40 \%)$ a été enregistrée dans la région du Plateau central. La région de la Boucle du Mouhoun a enregistré la sévérité moyenne la plus élevée qui est de 85,06 \pm $18,25 \%$ et la région du Nord une sévérité intermédiaire $(62,12 \pm 34,50)$.

La Figure 8 illustre le niveau moyen de sévérité des attaques des thrips sur les différents sites de production d'oignon. La sévérité la plus faible $(15,99 \pm 4,75 \%)$ a été enregistrée à Goué dans le Plateau central et la plus élevée $(99,61 \pm 0,88 \%)$ a été relevée à Nokuy dans la Boucle du Mouhoun.

Tableau 1: Densité, incidence et sévérité moyennes en fonction de la zone agro climatique. $\mathrm{N}=$ Nbre de parcelles. (ANOVA : test de Student-Newman-Keuls (S-N-K) au seuil de 5\%). S : significatif ; NS : non significatif.

\begin{tabular}{llll}
\hline $\begin{array}{l}\text { Zone agro } \\
\text { climatique }\end{array}$ & $\begin{array}{l}\text { Densité moyenne } \\
\left(\text { thrips au } \mathbf{~ m}^{2}\right)\end{array}$ & Incidence moyenne (en \%) & Sévérité moyenne (en \%) \\
\hline Sahélienne & $216 \pm 234$ a & $72,3 \pm 37,7$ a & $62,21 \pm 37,25$ a () \\
Nord soudanienne & $64 \pm 165 \mathrm{~b}$ & $63,6 \pm 26,7$ a & $52,18 \pm 26,34$ a () \\
Pr $>$ F & 0,037 & 0,45 & 0,38 \\
\hline Signification & $\mathrm{S}$ & NS & NS \\
\hline
\end{tabular}

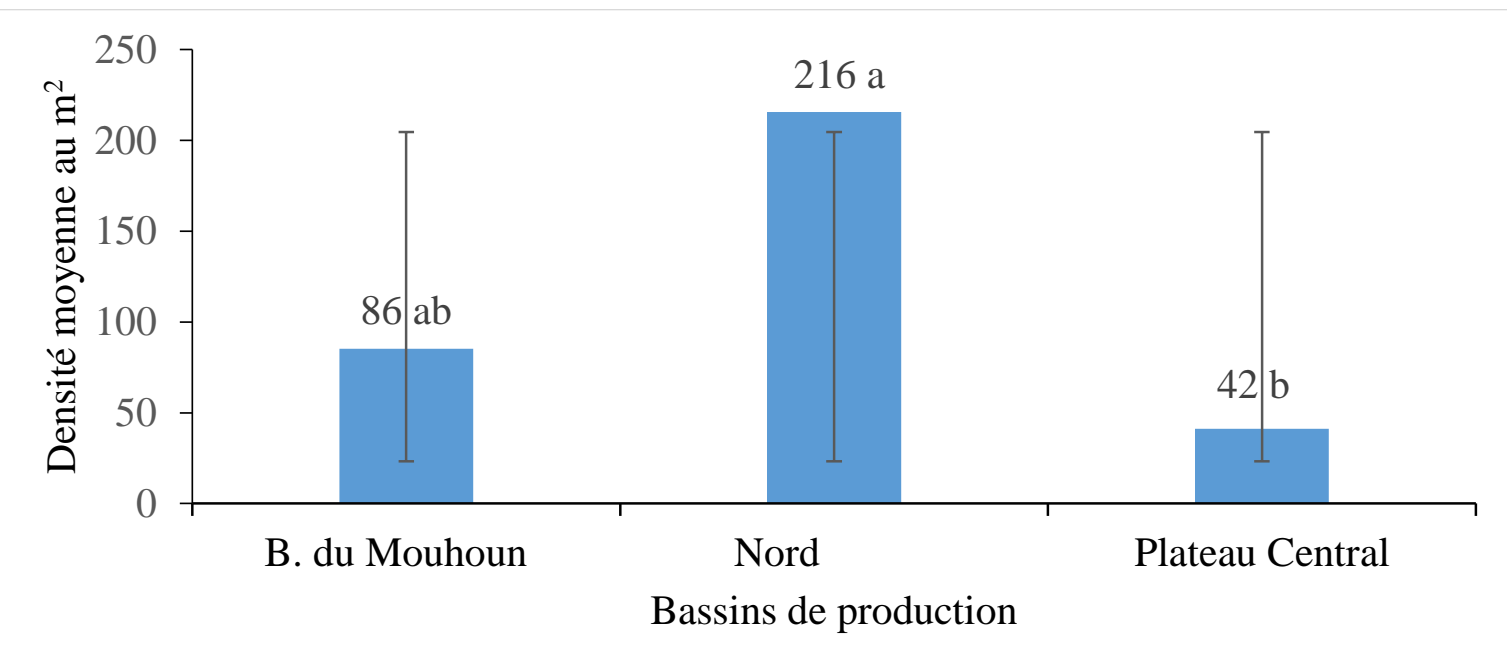

Figure 3: Densité moyenne des thrips au $\mathrm{m}^{2}$ en fonction du bassin de production. $\mathrm{N}=$ Nbre de parcelles. (ANOVA : test de Student-Newman-Keuls (S-N-K) au seuil de 5\%). 


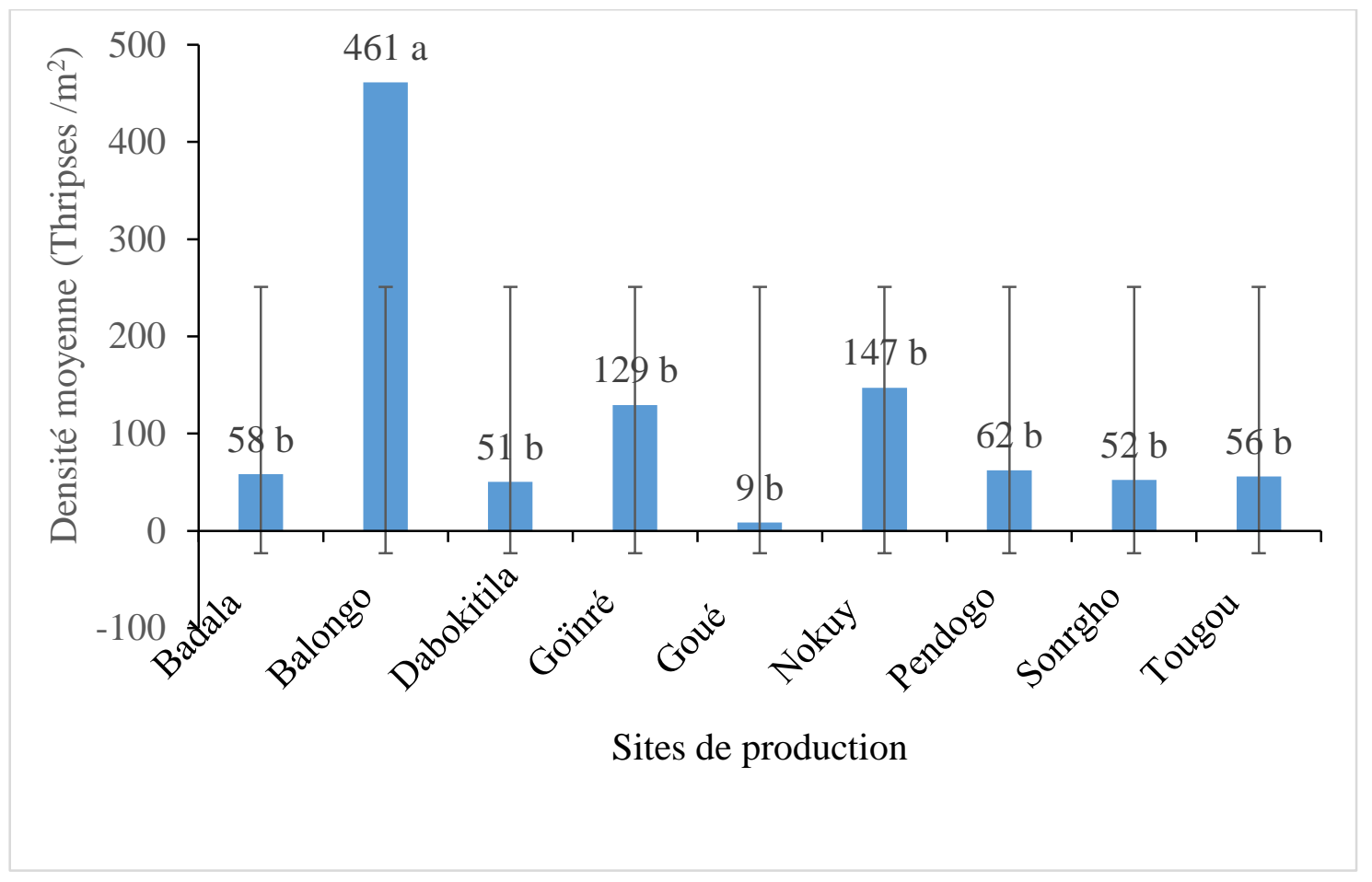

Figure 4: Densité moyenne de thrips au $\mathrm{m}^{2}$ en fonction du site de production. $\mathrm{N}=\mathrm{Nbre}$ de parcelles. (ANOVA : test de Student-Newman-Keuls (S-N-K) au seuil de 5\%).

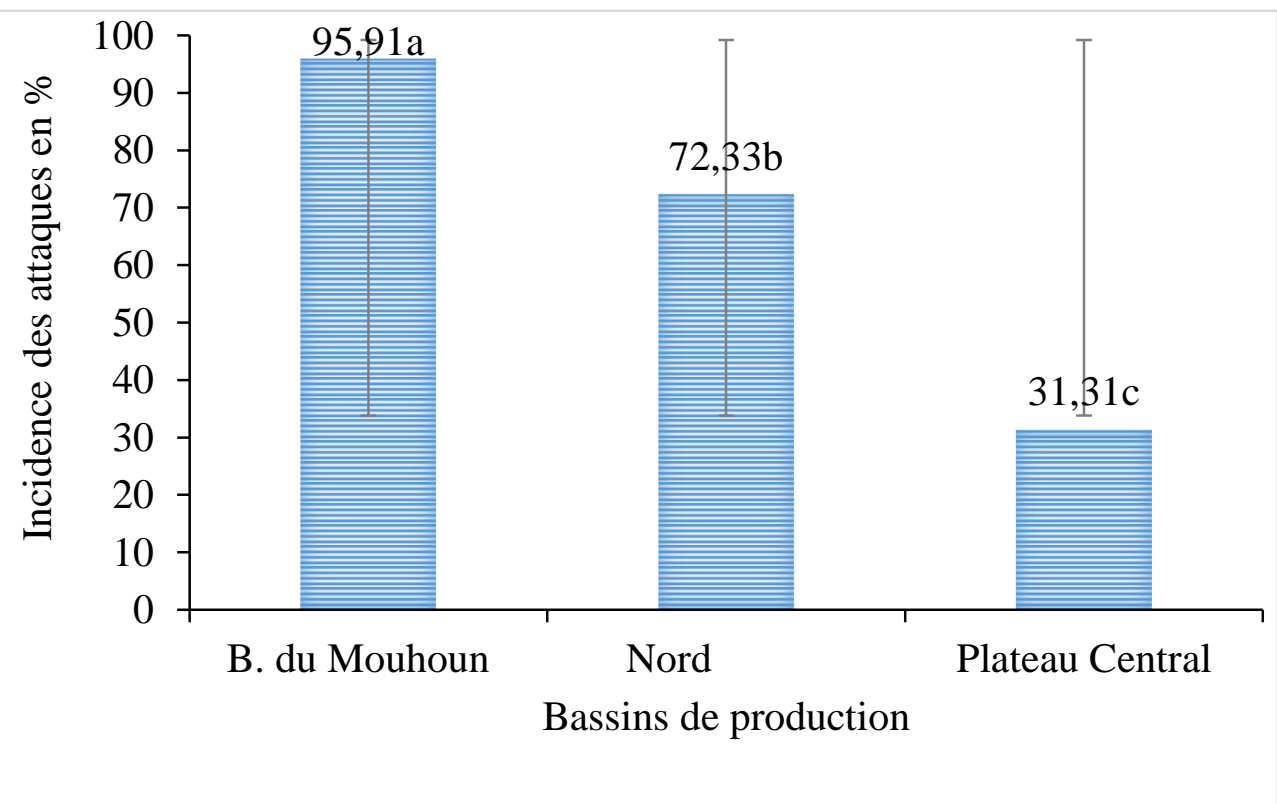

Figure 5: Niveau moyen de l'incidence des attaques des thrips en fonction du bassin de production. $\mathrm{N}=$ Nbre de parcelles. (ANOVA : test de Student-Newman-Keuls (S-N-K) au seuil de 5\%). 


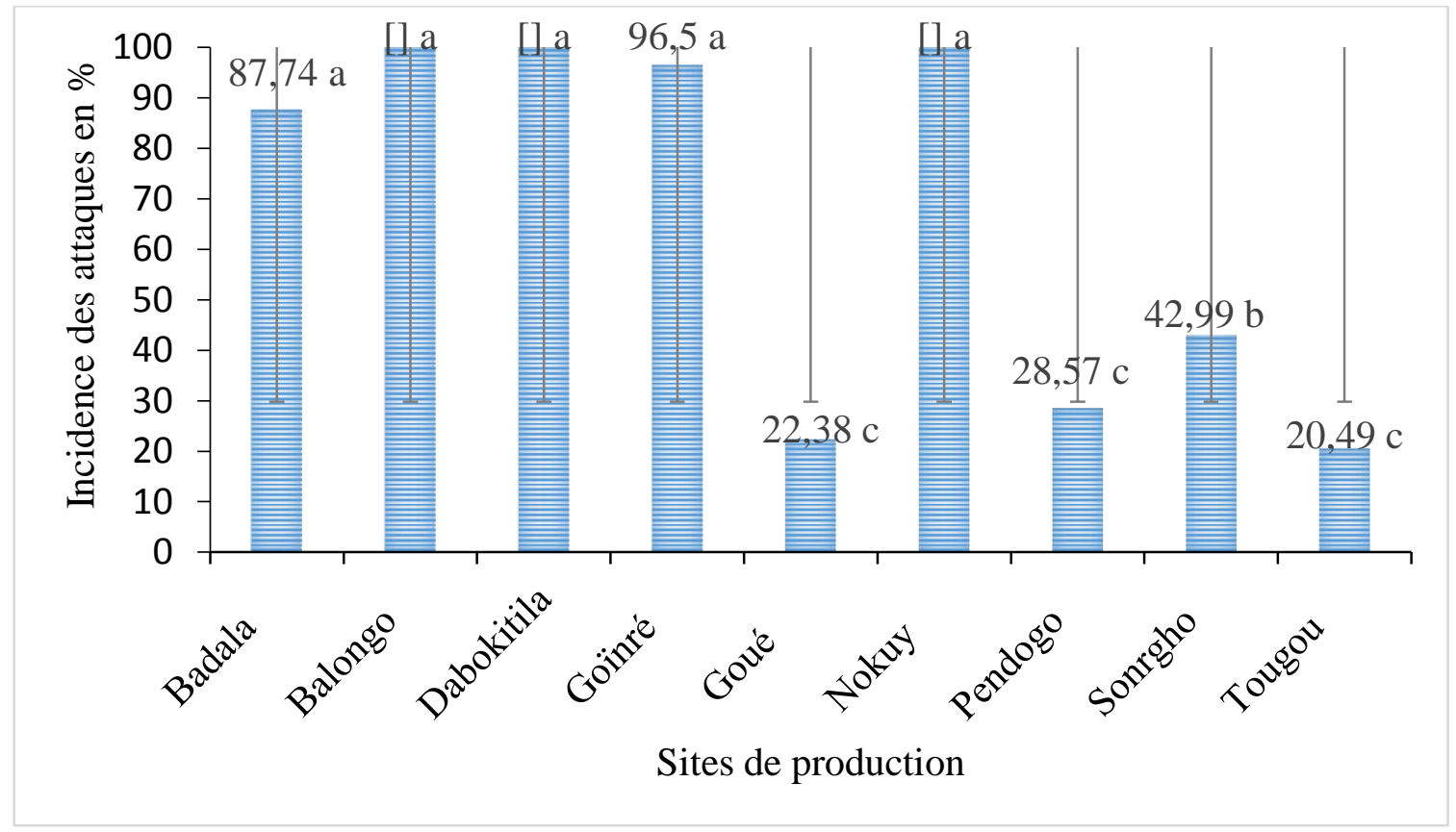

Figure 6: Incidences moyennes des attaques des thrips en fonction du site de production. $\mathrm{N}=\mathrm{Nbre}$ de parcelles. (ANOVA : test de Student-Newman-Keuls (S-N-K) au seuil de 5\%).

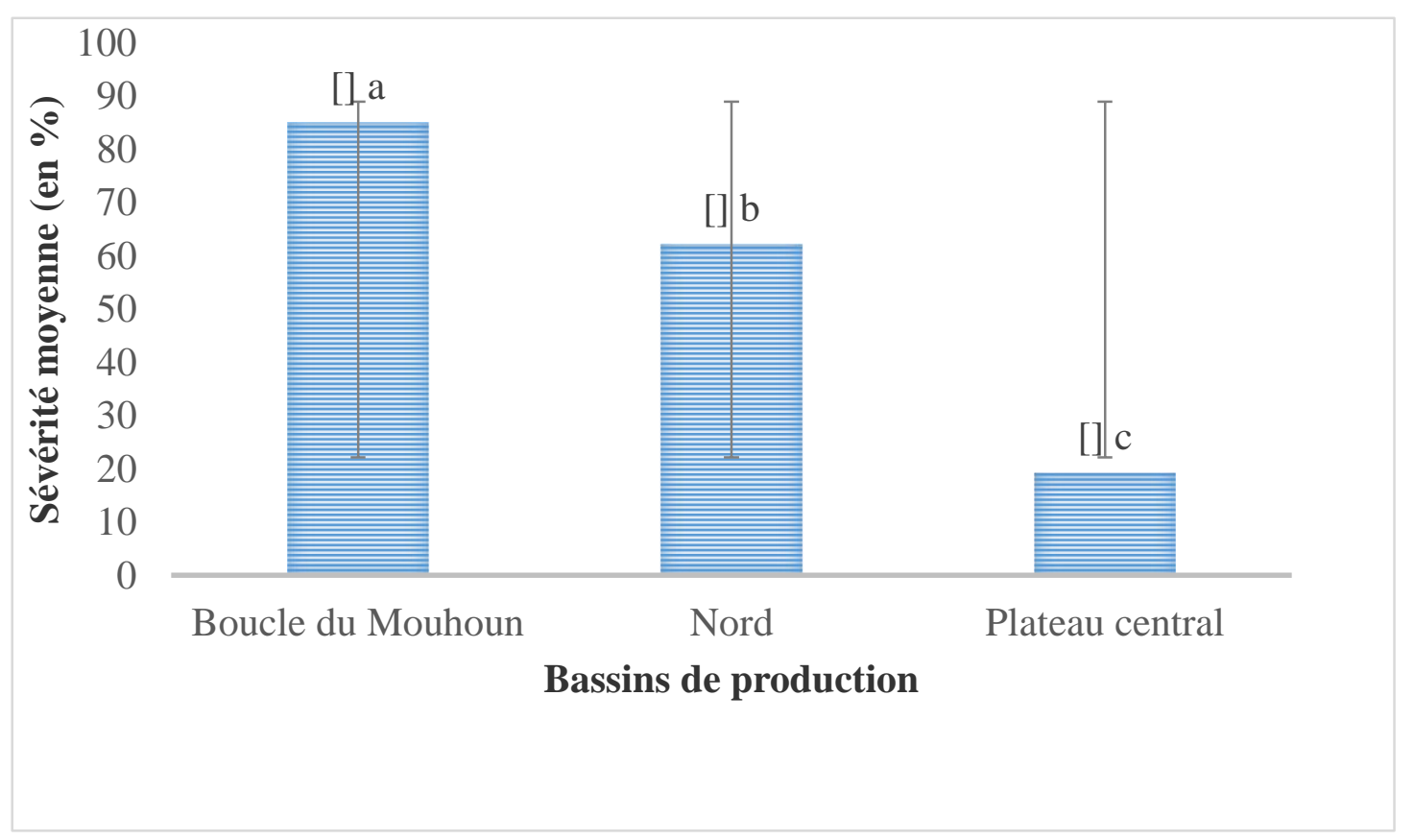

Figure 7: Sévérité moyenne des attaques du T. tabaci en fonction du bassin de production. $\mathrm{N}=$ Nbre de parcelles. (ANOVA : test de Student-Newman-Keuls (S-N-K) au seuil de 5\%). 


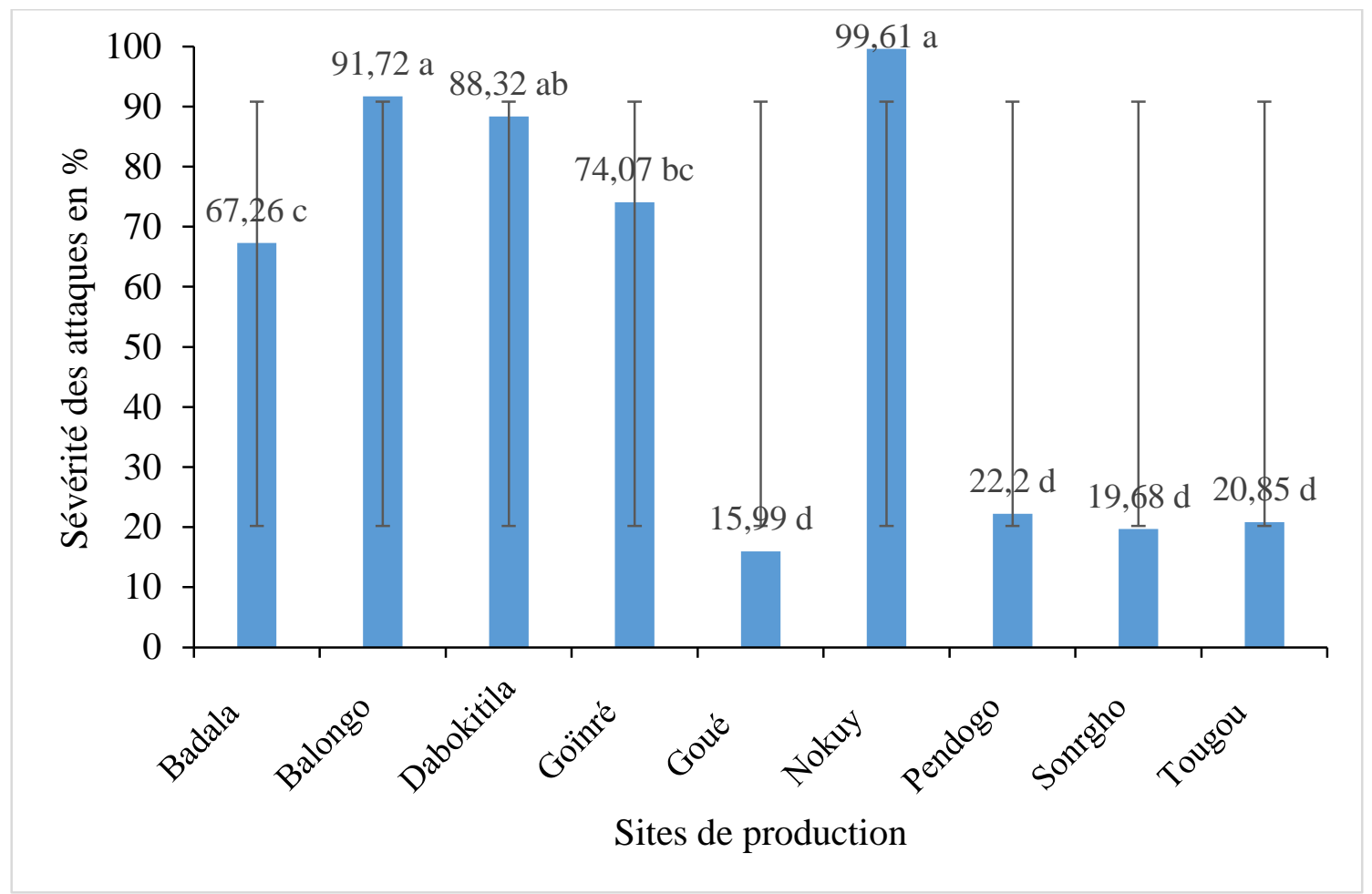

Figure 8: Sévérité moyenne des attaques de thrips en fonction du site de production d'oignon. $\mathrm{N}=$ Nbre de parcelles. (ANOVA : test de Student-Newman-Keuls (S-N-K) au seuil de 5\%).

\section{DISCUSSION}

La densité moyenne des thrips au $\mathrm{m}^{2}$ la plus élevée a été observée à Balongo. Cette forte densité pourrait s'expliquer par le mode de gestion des résidus de récolte sur ce site de production d'oignon. En effet, Balongo avec $80 \%$ de producteurs qui abandonnaient les résidus de culture au champ est le site qui abrite la proportion de maraichers la plus importante à qui est associée cette mauvaise pratique agricole. Blancard (2013) et Haougui et al. (2014), ont rapporté que l'abandon des résidus de récolte au champ favorise la multiplication, la dispersion et la survie des thrips. Ce même constat a été fait aussi par Soucy (sd). A l'opposé de Balongo, la densité de thrips au $\mathrm{m}^{2}$ la plus faible a été observée à Goué. Sur ce site, seulement $10 \%$ des producteurs enquêtés abandonnaient les résidus de récolte au champ et $70 \%$ pratiquaient l'irrigation par aspersion. Selon Haougui et al. (2014), l'irrigation par aspersion est une solution efficace pour perturber la population de thrips et finalement pour lutter contre ce ravageur. Aussi, Goué a reçu une pluie à deux jours de notre prospection sur ce site. Harris et al. (1936) ont remarqué une réduction importante des effectifs de T. tabaci sur l'oignon après des pluies et de la grêle. Bournier (1983) et Kirk (1997) ont rapporté que de fortes précipitations sont responsables de la destruction d'une grande partie des populations de thrips.

A l'échelle des bassins de production d'oignon, nous avons montré qu'il existait une différence de densité moyenne de thrips au $\mathrm{m}^{2}$ entre les régions du Nord et du Plateau central. Cette importante différence peut s'expliquer par la très forte densité de thrips sur le site de Balongo dans le Nord et la très faible densité à Goué dans le Plateau Central.

En ce qui concerne l'incidence moyenne au niveau des sites de production, la 
plus élevée a été observée à Balongo dans le Nord, à Dabokitila et à Nokuy dans la Boucle du Mouhoun. La plus faible densité a été observée à Tougou dans le Nord. La faible incidence des thrips observée à Tougou pourrait s'expliquer par les moyens de lutte utilisés contre les insectes. Tougou est le seul site où les producteurs n'ont pas utilisé d'insecticides chimiques pour protéger leur oignon. Les exploitants de ce site ont plutôt utilisé des insecticides biologiques (extraits de plantes) pour assurer des traitements préventifs contre les ravageurs. Ces producteurs ont également utilisé la cendre dont ils ont saupoudré les plantes d'oignon lorsque les premiers symptômes des attaques de thrips étaient aperçus dans quelques parcelles. Selon Soucy (sd), l'utilisation de la chaux hydratée diminue temporairement la population des thrips. Picault et al. (2018) ont quant à eux, rapporté que l'utilisation du kaolin exerçait aussi un effet significatif sur l'infestation des cultures de poireau par T. tabaci. En effet, le kaolin agit comme répulsif en créant un film qui n'est pas propice à l'alimentation et à la ponte des insectes piqueurs-suceurs. Aussi, sur ce site aucun producteur n'avait abandonné les résidus de récolte au champ.

Les résultats de l'analyse de variance portant sur les incidences moyennes dans les bassins de production ont révélé une différence significative entre les trois régions. La forte incidence de thrips observée dans la Boucle du Mouhoun pourrait s'expliquer par la manière dont les résidus de récolte étaient gérés (abandons aux champs élevés) par les producteurs, le mode d'irrigation pratiqué (irrigation à la raie) mais surtout par la période de prospection des sites dans cette région et le repiquage tardif de l'oignon dans certains sites. En effet, un peu plus de 50\% des producteurs enquêtés dans cette région abandonnaient leurs résidus de récolte au champ. Aussi, la prospection des sites de cette région a été effectuée en mi-avril, période de forte chaleur $\left(31,27{ }^{\circ} \mathrm{C}\right.$ de température moyenne). Anonyme (2019), a rapporté que les températures élevées favorisaient la pullulation des thrips. Sous un climat chaud, les générations se succèdent à un rythme très accéléré et il peut y avoir 12 à 15 générations chaque année a rapporté Lewis $(1973 ; 1997)$. Biobest (sd), a rapporté que si la température est suffisamment élevée, une femelle de thrips peut produire jusqu'à 200 descendants. En plus, la phase végétative de l'oignon a coïncidé avec la pullulation des thrips (mois d'avril) à cause du repiquage tardif (fin janvier- février) dans certains sites.

La sévérité moyenne la plus élevée a été observée à Nokuy et la plus faible a été enregistrée à Goué. En ce qui concerne les bassins de production, les résultats de l'analyse de variance ont révélé une différence significative entre les trois régions. La Boucle du Mouhoun a enregistré la sévérité la plus élevée. Cette région est suivie par le Nord. La plus faible a été observée dans la région du Plateau central. Cette différence pourrait s'expliquer par les pratiques culturales utilisées (système d'irrigation, gestion des résidus de récolte, moyens de lutte), mais aussi par le nonrespect du calendrier cultural (repiquage tardif de l'oignon).

\section{Conclusion}

Les résultats de la présente étude, permettent de conclure que les pratiques agricoles influencent considérablement la prolifération de T. tabaci, principal ravageur de Allium cepa. La densité moyenne des thrips au $\mathrm{m}^{2}$ la plus élevée a été observée sur ce site avec 461,4 thrips au $\mathrm{m}^{2}$ et celle la plus faible $(8,6)$ a été observée à Goué. A l'échelle des bassins de production d'oignon, il a été mis en évidence une différence de densité moyenne de thrips au $\mathrm{m}^{2}$ entre les régions du Nord $(215,53)$ et du Plateau central $(41,13)$. En ce qui concerne l'incidence moyenne au niveau des sites de production, la plus élevée $(100 \%)$ a été observée à Balongo dans le Nord, à Dabokitila et à Nokuy dans la Boucle du Mouhoun. La plus faible $(20,49 \%)$ a été observée à Tougou dans le Nord où les exploitants de ce site ont utilisé des insecticides biologiques pour assurer des traitements préventifs contre les ravageurs. L'étude a révélé une différence significative entre les trois bassins de production en ce qui concerne la sévérité des 
attaques de thrips. La boucle du Mouhoun a enregistré la sévérité la plus élevée $(85,06 \%)$. Elle est suivie par le Nord $(62,21 \%)$ et la plus faible $(19,29 \%)$ a été observée dans la région du Plateau central.

Cette étude pourrait contribuer à une plus grande connaissance de l'insecte et de ses effets néfastes sur l'oignon, principale culture maraichère du Burkina Faso. Ainsi, à la lumière de ce qui précède, nous recommandons aux différents partenaires des sensibilisations et des formations des acteurs de la production de l'oignon sur la connaissance de T. tabaci, de ses dégâts et des moyens de lutte efficace contre cet insecte, principal ravageur de l'oignon.

\section{CONFLIT D'INTERETS}

Aucun conflit d'intérêts potentiel n'a été signalé par les auteurs.

\section{CONTRIBUTIONS DES AUTEURS}

AS a préparé le protocole, $\mathrm{SN}, \mathrm{BBB}$, $\mathrm{KN}$ et MWS l'ont amendé. AS a réalisé la collecte des données sur le terrain avec l'appui de SN. MWS, BBB et SN ont aidé AS à exploiter les données collectées et à préparer le manuscrit. Tous les autres auteurs ont lu, amendé et approuvé le manuscrit.

\section{REMERCIEMENTS}

Ce travail est le fruit des efforts de plusieurs personnes dont nous tenons à remercier. Nous remercions les autorités du CAP Matourkou, tous les partenaires du projet PRoduIRE, les producteurs/trices des différents sites couverts par l'étude et toute personne qui a contribué à la concrétisation de cette étude.

\section{REFERENCES}

Abdourahamane HM, Baoua I, Lawali S, Tamò M, Amadou L, Mahamane S, Pittendrigh B. 2019. Essai comparatif de l'utilisation des extraits du Neem et du virus entomopathogène MaviNPV dans la gestion des insectes ravageurs du niébé en milieu paysan au Niger. Int. J. Biol.
Chem. Sci., 13(2): 950-961. DOI: https://dx.doi.org/10.4314/ijbcs.v13i2.30 Abdoulaye ZO, Baoua I, Amadou L, Tamò M, Pittendrigh BR. 2019. Les contraintes entomologiques de la culture du niébé et leur mode de gestion par les producteurs dans les régions de Maradi et Zinder au Niger. Int. J. Biol. Chem. Sci., 13(3): 1286-1299.

DOI: https://dx.doi.org/10.4314/ijbcs.v13i2.30

Ablo-Elyour MAK, Abdel-Hafez SII, AbdelRahim IR. 2014. Isolation of Trichoderma and Evaluation of their Antagonistic Potential against Alternaria porri. Journal of Phytopathology, 162(9): 567-574. DOI: https://doi.org/10.1111/jph.12228

Agbahoungba S, Karungi J, Odong TL, Badji A, Sadik K and, Ruhaihayo PR. 2017. Stability and extent of resistance of cowpea lines to flower bud thrips in Uganda. African Crop Science Journal, 25(1) : $\quad 1-24$. DOI: http://dx.doi.org/10.4314/acsj.v25i1.1

Anonyme. 2019. Thrips tabaci, redoutable ennemi de l'oignon. https://www.bayeragri.fr/culture/thrips-tabaci-redoutableennemi- de- l'oignon_2545/ consulté : le $19 / 02 / 2019$.

Biao F, Afouda L, Kone D. 2019. Prévalence des maladies virales du piment (Capsicum spp.) et perception des producteurs dans la commune de Malanville au Nord-Bénin. Int. J. Biol. Chem. Sci., 13(1): 166-177. DOI: https://dx.doi.org/10.4314/ijbcs.v13i1.14

Blancard D. 2013. Melon-Thrips, ephytia.inra.fr consulté le 15/09/2019.

Bournier A. 1983. Thysanoptères de France. VII. Bulletin de la Société Entomologique de France, 88: 1-9.

Clinique des Plantes. 2018. Fiche technique du Thrips tabaci. 2p.

Dabiré FS. 2016. Etude de l'incidence de la pourriture basale de l'oignon (Allium cepa L.) dans la Vallée du Sourou et l'évaluation de la résistance/tolérance de onze variétés vis-à-vis de la maladie. Mémoire d'Ingénieur du développement 
rural, Université Polytechnique de Bobo Dioulasso, Burkina Faso, p.60.

DPSSA. 2011. Phase 2, RGA 2006-2010. Rapport général du module maraîchage. Ministère de l'Agriculture et de l'Hydraulique, Burkina Faso, p.318

Egho EO. 2011. Management of major field insect pests and yield of cowpea (Vigna unguiculata $(\mathrm{L})$ walp) under calendar and monitored application of synthetic chemicals in Asaba, southern Nigeria. Am. J. Sci. Ind. Res., 2(4): 592-602. DOI:10.5251/ajsir.2011.2.4.592.60

Haougui A, Delmas P, Kimba A. 2014. Formation sur les producteurs et les structures d'appui conseil sur les maladies et ravageurs de l'oignon p.15.

Harris HM, Drake CJ, Tate HD. 1936. Observations of the onion thrips. Iowa State College Journal Science, 10: 155172.

Issoufou OH, Boubacar S, Adam T, Boubacar Y. 2017. Identification des insectes, parasites et évaluation économique de leurs pertes en graines sur les variétés améliorées et locale de niébé en milieu paysan à Karma (Niger). Int. J. Biol. Chem. Sci., 11(2): 694-706. DOI: http://dx.doi.org/10.4314/ijbcs.v11i2.13

Kirk WDJ. 1997. Distribution, abundance and population dynamics. In Thrips as Crop Pests, Lewis T (Ed.). CAB international : Wallingford, UK; 217-257.

Leblanc M. 2010. Le thrips de l'oignon : un insecte difficile à réprimer. Ed. Agriculture, Pêcherie et Alimentation, Québec, p.6.

Lewis T. 1973. Thrips, their Biology, Ecology and Economic Importance. Academic Press: New York.

Lewis T. 1997. Thrips as Crop Pests. CAB. Int. Institute of Arable Crops Research: Rothamsted, Harpenden, Hertfordshire, UK.
Liao CT, Lin CS. 2000. Occurrence of legume pod borer Maruca testulalis (Geyer) (Lepidoptera: Pyralidae) on cowpea Vigna unguiculata (L.) Walp and its insecticides application trial. Plant Pro. Bull, 42: 213-222.

Picault S, Quennesson S, Roy G, Davy M, Torres M, Ginez A, Schoeny A, Lambion Lavigne JD, Bouvard D. 2018. Utilisation de plantes de service et de kaolin pour gérer les populations de pucerons et de thrips en cultures légumières. Innovations Agronomiques, INRA, 63 : 385-397. DOI :

dx.doi.org/10.15454/1.51911935844305 $71 \mathrm{E} 12$

Programme de Développement des Cultures Fruitières et Légumières (PDCEL). 2017. Situation de références de la production fruitière et légumière. Phase 2018-2020.

Prota. 2004. Ressources Végétales de l'Afrique Tropicale (vol. 2). Légumes.. Grubben GJH, Denton OA (Éds). Fondation PROTA Backhuys, CTA: Wageningen; 737.

Razi S. 2017. Etude éco-biologique des thrips de la région de Biskra, Algérie. Thèse de doctorat en sciences agronomiques. Université de Biskra, p. 145.

Son G, Kiogo R, Yé SG. 2016. Analyse des systèmes de production de l'oignon bulbe autour du barrage de Goinré dans la province du Yatenga au Nord du Burkina Faso. Int. J. Biol. Chem. Sci., 10(3): 11731183.

DOI: http://dx.doi.org/10.4314/ijbcs.v10i3.22

Villeneuve F, Thicoipe JP, Legrand M, Bosc JP. 1999. Le Thrips du poireau. Comment raisonner les interventions? Quelles stratégies? Infos Ctifl-150: 44-49. 